\title{
Distributed Object Implementations for Interactive Applications *
}

\author{
Vijaykumar Krishnaswamy, Ivan B. Ganev, Jaideep M. Dharap, and \\ Mustaque Ahamad \\ College of Computing, Georgia Institute of Technology, Atlanta, GA 30332 \\ $\{\mathrm{kv}$, ganev, jaideep, mustaq $\} @ c c$.gatech.edu
}

\begin{abstract}
As computers become pervasive in the home and community and homes become better connected, new applications will be deployed over the Internet. Interactive Distributed Applications involve users in multiple locations, across a wide area network, who interact and cooperate by manipulating shared objects. A timely response to user actions, which can potentially update the state of the objects, is an important requirement of interactive applications. Because of the inherent heterogeneity of the environment, distributed applications are built using technologies like distributed objects. Central server based implementations of distributed objects cannot meet the response time needs of interactive users because invocations are always subject to communication latencies. Our approach is to extend these technologies with aggressive caching and replication mechanisms to provide interactive response time and to improve scalability. A flexible caching framework is presented, where objects can be cached in an application specific manner. It provides multiple consistency protocols that enable tradeoffs between the consistency of a cached object's state at a particular client, and the communication resources available to the client. At runtime, clients can specify their consistency requirements via a Quality of Service specification interface that is meaningful at the application level. This paper presents the caching framework, its implementation and some preliminary performance results.
\end{abstract}

Keywords: Remote Method Invocation(RMI), Caching, Consistency Protocols, Timeliness, Quality of Service.

\section{Introduction}

As computers become pervasive in the home and community and homes become better connected, a new class of applications will emerge in the wide-area distributed computing environment. We consider applications that we call interactive distributed applications. These applications involve users in multiple locations who interact and cooperate with each other by manipulating shared

\footnotetext{
* This work was supported in part by NSF grants CCR-961937 and a grant from the Southeastern Universities Research Association.
} 
objects. Examples of such applications include collaborative design, distributed games, and group oriented educational applications.

The system support that will enable interactive distributed applications must address a number of challenges. First, because the applications are interactive, it is necessary to provide quick response to a user's action even when the remote users with whom he or she is interacting with are connected by a high latency communication network. Users must also observe remote actions in a timely fashion, and the timeliness requirements could vary across users due to differences in available resources, or because of the differing roles users play in the application. Finally, users must have a consistent view of the shared objects that are manipulated by them.

Interactive distributed applications can be built using technologies such as distributed objects (e.g, Java RMI, CORBA or DCOM). Although these technologies are attractive for building such applications in heterogeneous environments, they require that shared objects be implemented by common servers, and users must access such an object by remotely invoking it at the server node. Such centralized servers are undesirable because response time for user actions that manipulate the objects cannot be independent of the communication latencies in the system. Although proxy technology that allows a client site to cache an object has been explored[24], many issues that arise when dynamically changing state is cached have not been addressed. We are exploring an approach that retains the ease of programming benefits of distributed objects while providing interactive response time to the invocations made to them. This is done by replication and caching of object state where it is accessed. Consistency requirements arise across the multiple copies that are created when replication and caching are employed. We develop a quality of service (QoS) interface that allows applications to specify their consistency needs. For example, a client can specify timeliness requirements to ensure that it learns of a remote update to a cached object within a certain time period after the write is done.

We present a framework for caching Java distributed objects at client sites. Since we want applications to control the level of consistency of a cached object via a simple QoS interface, we chose BBN's Quality Object (QuO) framework[28] to specify shared state QoS metrics that are are meaningful at the application level. The following are the primary contributions of the paper.

1. We develop a framework that allows clients to invoke cached objects transparently. If a user action results in the invocation of one or more objects, often their cached copies can be invoked and hence response time independent of communication latencies can be provided. The clients only need to specify their consistency needs for the cached object copies, which is done at a high level using QuO's contract object facility.

2. We implement consistency protocols that are particularly well-suited for a heterogeneous environment. In particular, they offer consistency vs. resource usage tradeoffs, and different clients may request different levels of consistency. 
3. We develop a prototype system and use it to evaluate the costs of consistency protocols. Using the prototype, we show that invoking a locally cached copy is fifty times cheaper than a remote invocation at the server even in a local area network. We also evaluate the impact of varying timeliness based consistency on the response time of object invocation with a synthetic workload for interactive applications.

Section 2 describes an interactive distributed application and some interesting properties of such applications. Section 3 presents a brief overview of the system architecture. The consistency protocols and their implementation in the caching framework is described in Sect. 4. We present performance results in Sect. 5. Related work is discussed in Sect. 6 and the paper is concluded in Sect. 7.

\section{Interactive Applications}

Interactive applications process user input and respond to user actions on a continuous basis. We consider distributed interactive applications that involve several users in different locations. The actions issued by one user could impact other users and hence their actions. Many such distributed interactive application scenarios can be developed easily. We briefly describe the AquaMoose[6] system that is currently being developed at the Georgia Institute of Technology. AquaMoose supports an online community of children interested in educational activities. These children can be geographically distributed and can share and manipulate a virtual world representing an ocean. A user can create various entities in the world and entities created by different users may have rich interactions. For example, two fish can race in trajectories defined by their creators and the bigger fish may eat the smaller one. The virtual world visualization at each user is driven by the state of the entities in the world (e.g., fish) including their location and direction of movement. The entity state changes dynamically as the virtual world evolves.

A closer study of AquaMoose and other such applications reveals some very interesting properties. These applications have state that is highly dynamic. Also, for these applications to perform well, the response time to the user actions should be bounded. For example, a delay of more than $100 \mathrm{~ms}$ in a direct manipulation interface is perceptible. As the delay for these user actions increases, the user satisfaction with these applications worsens. If the participants in these applications are connected via a wide area heterogenous network, network latencies could be much larger than this threshold. One approach for developing such applications is to maintain a replica of the shared application state on the local machine and keep it consistent with the replicas at other participating sites by using consistency protocols. This way invocations made by user actions can be executed with the local copy. Also, different user actions may require different levels of consistency for their replicas. For instance, in the AquaMoose example, if two fish are far away from each other and are controlled by two geographically separated users, the updates made to their attributes (e.g. locations) can 
be disseminated relatively slowly to the other sites. But if these fish are in close proximity, then the updates made to their locations should be quickly transferred to the other site for acceptable execution behavior of the application. In the following sections we will explore the system support for developing such highly interactive and dynamic distributed applications.

\section{System Architecture}

In a distributed object system, invocation to a remote object requires communication with the server that implements the object. To provide acceptable performance for user actions in interactive applications, it is desirable that latencies associated with method invocations be minimized. In wide area systems, a major portion of the invocation time can be attributed to network latencies. This overhead can be avoided by locally caching the state of the objects used for building these applications. Caching can be effective in such applications because of two reasons. First, GUI based visualization of the application is driven by the state of the shared objects. Hence, their state is frequently accessed. Second, updates to cached object state can be disseminated periodically, depending on the consistency requirements of the applications. For example, in the AquaMoose application, cached state (or computed state using techniques such as dead reckoning[13]) can be used until a new update for a fish's position is received.

We have developed a caching framework for distributed objects that can transparently cache the objects at the clients that invoke them. The consistency requirements can differ depending on application needs and where the application is deployed. The framework that we have developed addresses this by providing facilities for dynamic specification of consistency protocols and its parameters. The Quality Objects $(\mathrm{QuO})[28]$ project offers a framework for creating applications that adapt to different Quality of Services (QoS) offered by the underlying system. Rather than developing a new QoS specification interface from ground up for our caching framework., we chose to use $\mathrm{QuO}$ to explore shared state QoS. In the following section we will briefly discuss the support that we have added to $\mathrm{QuO}$ for specifying and maintaining the QoS of an object's cached state.

\subsection{QuO Framework}

$\mathrm{QuO}$ is a framework that has been developed to support distributed applications with $\mathrm{QoS}$ requirements. $\mathrm{QuO}$ provides the ability to specify, monitor, and control QoS in an application. In a traditional CORBA application, a client makes a method call on a remote object through its functional interface. The call is processed by an object request broker (ORB) on the client's host, delivered to an ORB on the object's host, and executed by the remote object. The client sees it strictly as a functional method call. A QuO application adds additional steps to this process for QoS evaluation. As shown in Fig. 1, all QuO application consists of the following additional components. 
- A QoS contract between the client and the object. This specifies the level of service desired by the client, the level of service the object expects to provide, operating regions indicating possible measured QoS, and actions to take when the level of QoS changes.

- A smart delegate of the remote object. The delegate provides a functional interface identical to the remote object, but can trigger contract evaluation upon each method call and return. The QoS developer can provide alternative behaviors and a dispatch statement based on the current state of the contract.

- System condition objects interface between the contract and resources, objects and ORBs in the system. These are used to measure and control QoS. They are shown as the polygons with syscond label in Fig. 1.

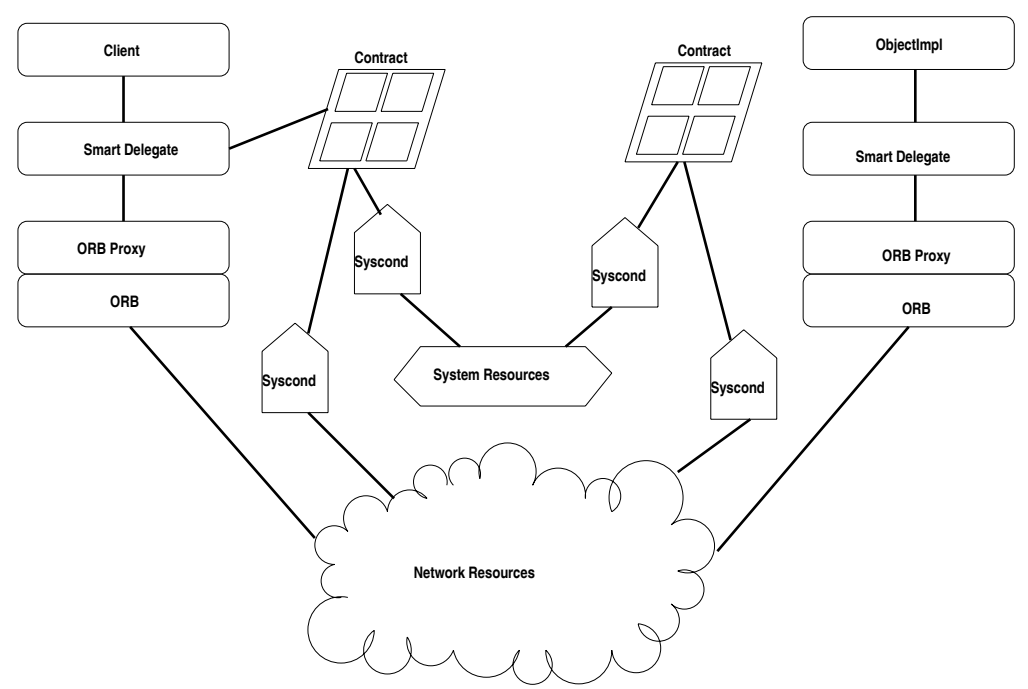

Fig. 1. The Quality Object Framework

A suite of Quality Description Languages (QDL) for describing contracts, system condition objects and the adaptive behavior of objects and delegates is provided by the $\mathrm{QuO}$ system. QDL consists of a Contract Description Language (CDL) and a Structure Description Language (SDL). CDL is used to describe the QoS contract between a client and an object. SDL describes the internal structure of delegate's implementations, such as implementation alternatives, and the adaptive behavior of object delegates. The object delegate generator creates client-side and server-side object delegate code from SDL, CDL, and IDL code. When a client calls a remote method, the call is passed to the object's smart delegate instead. The delegate can trigger contract evaluation, which accesses the current values of system condition objects. More details on QuO's architecture can be found in [28]. 
We show how QuO's contract facility can be used to manage the QoS of the shared state of an interactive application such an AquaMoose. The entities (fish) in the application are exclusively owned by users who create them. The attributes of these entities are only changed by the owners, while other users who are in the vicinity of these entities in the virtual world would like to observe these changes. Thus, one set of users mostly read the shared state of the object while the other one actively modifies it. Hence one set of users (i.e., the owners) would like to have active control of the object, while passive reading would be fine with the rest. These QoS requirements can be mapped onto the QuO's quality of service specification interface (i.e, the contract) as shown in the Fig. 2. A user who modifies the state of a shared object negotiates for the ActiveUser QoS region. PassiveUser region can be negotiated for by a user who mostly reads the state of the shared object.

A simple contract CacheStateContract, specifying the QoS regions of operation for the example application is shown in Fig. 2. The different QoS levels of operation are specified as regions in the contract. This contract is used to continuously monitor the current state of a cached object. CacheStateContract has several variable declarations. The variable ClientExpectedStaleness is used to specify the current timeliness QoS required by the user. ClientCallback is a handle to the callback object. This will be invoked whenever there is a discrepancy between the requested QoS and available QoS. CacheMonitor object is used to monitor the attributes of the caching framework that are of interest to the clients.

As shown in Fig. 2, the contract is divided into a series of regions. The users specify the desired region of operation through the variable ClientExpectedStaleness (different users can choose different regions).

In this particular contract example, the negotiated regions are ActiveUser and PassiveUser. A writer can choose the ActiveUser region while the readers can choose PassiveUser as their QoS region of operation. As seen from the sample code fragment in Fig. 2, the specification of a negotiated region triggers a sequence of events. For example, if the user negotiates for the ActiveUser region, then the transition any $->$ ActiveUser invokes the setProtocol method in the CacheMonitor object. This sets the consistency policy to the one that guarantees immediate dissemination of the new object values. Transition into PassiveUser region will also trigger events as in the ActiveUser region.

The available QoS for a resource at a given time is represented by a reality region. The QoS in a negotiated region can transition between the reality regions. In our example, the reality regions are Xclusive, Shared and Stale for the ActiveUser region. They correspond to the state in which the object is currently cached. Green, Orange and Red are the reality regions for the PassiveUser region. They indicate current age (potential staleness) of the cached object. Transition to any reality region triggers handlers in the callback object, which can be used to inform the client application. 


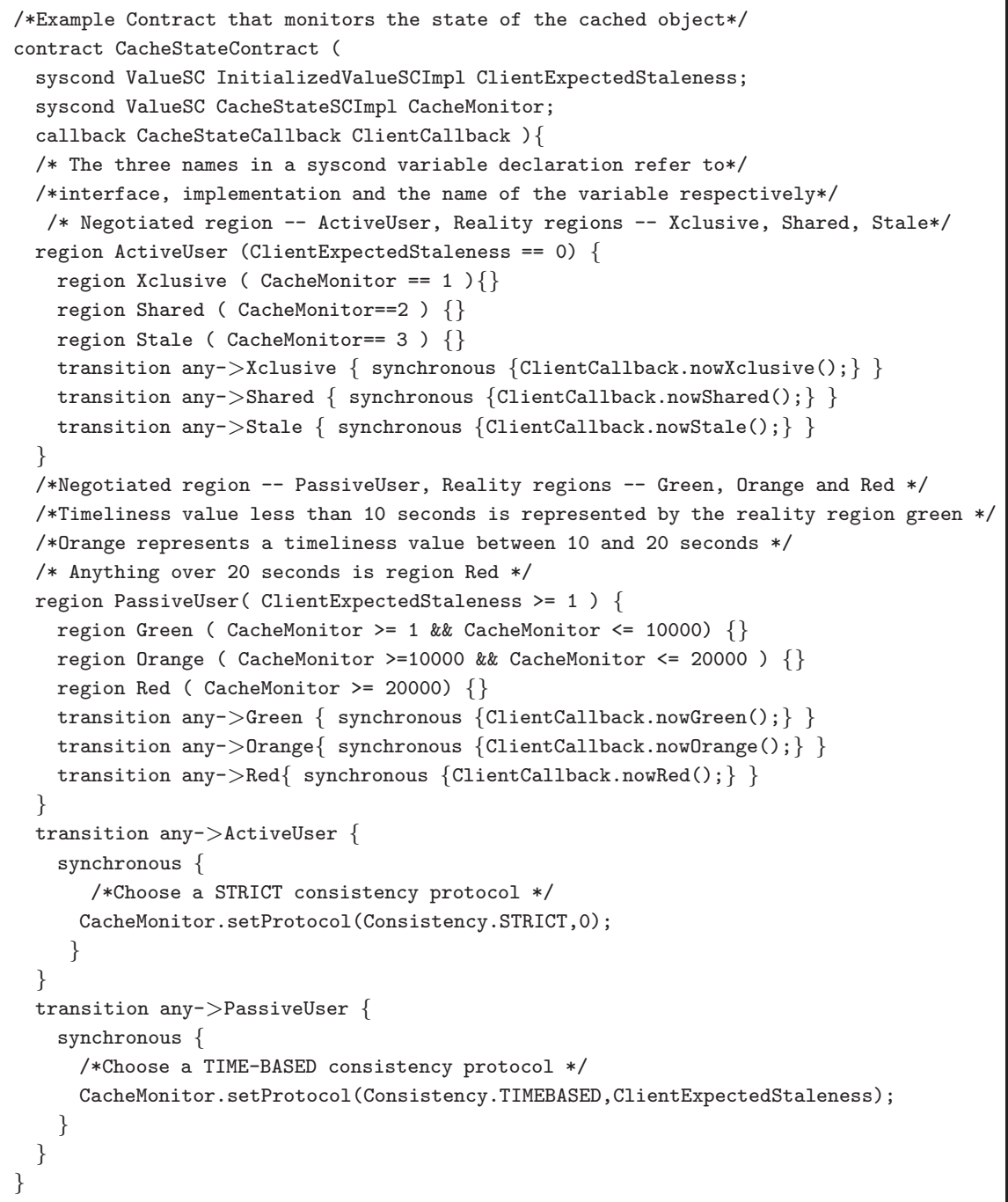

Fig. 2. A simple contract that exports the current state of the cached object to an application defined Callback object 


\subsection{Adding Caching to the QuO Framework}

In order to cache objects locally at client sites and provide consistency guarantees defined by the contract, the framework should address several important issues.

- How and when to cache an object? It should be possible to enable and disable caching dynamically based on locality of access and resource availability.

- How does the system guarantee the consistency QoS requirements of an application? How are the QoS levels associated with the appropriate consistency protocol and its parameters such as the timeliness threshold?

- The consistency actions executed by the protocols depend on the type of access, i.e., if an invocation results in reading of an object's state or the state is also updated. How is read/write access information for the object member functions inferred?

The caching framework developed by us tries to address these issues. Our current prototype has been developed in Java. Some of the objects that make up the caching framework on the server and client sides are shown in Fig. 3.

The caching subsystem on the client side consists of a CConsistencyObject which is responsible for maintaining the consistency of a cached implementation or implementations. The policy for the CConsistencyObject can be specified through a contract. The caching framework is accessed through SmartDelegate that is specifically created by QuO's stub generators for caching purposes. The SmartDelegate has two references to the remote object. The first one is a direct reference to the cached implementation. This is used whenever an invocation is executed with the locally cached implementation. The second one is a Java RMI remote object interface to the actual object implementation at the server. This is used to invoke the object at the remote location when caching is disabled. Assume that objects $O_{1}, O_{2}, \ldots, O_{n}$ are currently cached at the client. The objects, $\mathrm{RW}_{1}, \mathrm{RW_{2 }}, \ldots, \mathrm{RW} \mathrm{W}_{n}$ in Fig. 3 have the read/write access information for all the member functions of the implementations $0_{1}, 0_{2}, \ldots, 0_{n}$. These objects are generated from compile time tags associated with method definitions in the implementations. They are transferred along with object definitions to a client's address space, when the objects are cached for the first time at the client. The caching subsystem has a TransportObject which is used for communication with other clients and the server.

The server side of the framework consists of a SConsistencyObject. This orders the invocations that take place at the server with the ones that are executed with cached copies at client sites. Further, it serves as a rendezvous point for incoming clients, providing them with information to setup their caching framework. If the consistency policy happens to be server based, then SConsistencyObject plays a more active role in keeping the client copies consistent. The ServerDelegate redirects all the direct invocations made on the implementation at the server to SConsistencyObject, thus ensuring consistency when both cached and non-cached invocations are executed. RemoteClassLoader is used by the framework to remotely load the definitions of the implementations $\mathrm{O}_{1}, \mathrm{O}_{2}, \ldots, \mathrm{O}_{n}$, the read-write access information objects $\mathrm{RW}_{1}, \mathrm{RW}_{2}, \ldots, \mathrm{RW}_{n}$, 
and other objects that are referred to by the implementations in the server process. RemoteClassServer is the server side component that serves the class definition requests from the RemoteClassLoader.

The following are sequence of actions that are performed when an invocation o.m(), is made on the cacheable object, $o$.

1. The application invokes the method $m()$ in the SmartDelegate.

2. $\mathrm{QuO}$ semantics dictate a pre-method and a post-method evaluation of the contract. The delegate does a pre-method evaluation of the contract object to determine the current QoS region of operation. This can be used to determine the current state of the cached object, staleness value, ownership etc.

3. The contract checks with the system condition objects to determine the status of the cached object and if necessary, it also communicates with the callback object.

4. The delegate consults the consistency object to ensure that the cached object is in a consistent state with respect to the invocation. An invocation on the object can either read or modify the object state. Invocation mode can hence be either read or a write. Objects can be cached in shared or in exclusive mode. If the object is not valid or if the invocation mode does not match with the current mode in which the copy is cached, then the delegate asks the consistency object to perform the necessary consistency actions to bring the cached implementation to a consistent state. It also temporarily locks the implementation for the duration of the call thus providing method level atomicity.

5. The delegate invokes the method on the local object copy.

6. The delegate then makes a request to the the consistency object to free up the resources held by the call, to allow any pending consistency actions to be executed.

7. During the post-method evaluation, the delegate once again communicates with the contract to determine the active region.

8. The contract probes the system condition objects for new values. This can be useful for post method operations like starting new consistency actions in the background without blocking the application.

9. The delegate finally returns the results to the application.

\section{Consistency Protocols}

Consistency protocols ensure that client invocations are executed with local object copies that have consistent state. The consistency of a cached copy of an object can be defined along two dimensions, namely orderliness and timeliness. The orderliness property specifies how updates to the object done at various nodes are ordered and viewed by read operations. For the convergence of an object's state (e.g., a unique final state of the object is obtained after the execution of the invocations), it is essential that all writes to it are ordered. Weaker 


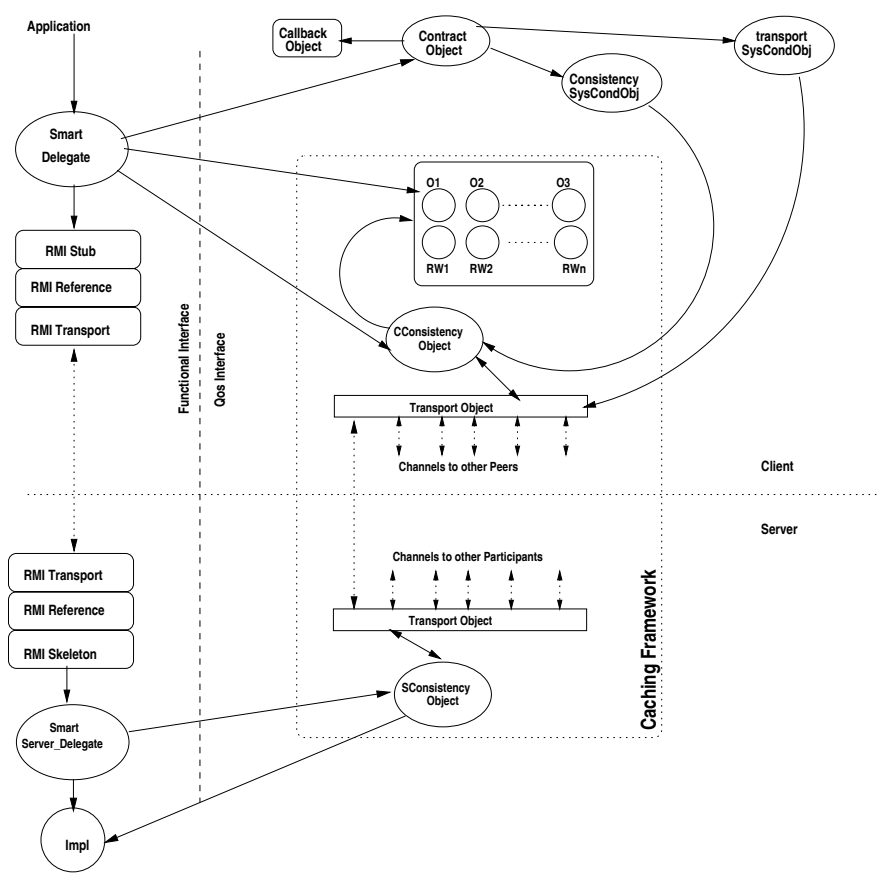

Fig. 3. The Caching Framework in the context of $\mathrm{QuO}$

orderings are possible (e.g., causal[2]) when concurrent writes are rare or other mechanisms can be used to fix divergent object state.

The timeliness property specifies the time interval after which an update to an object must become visible at sites that are caching it. By controlling the timeliness interval, updates can be propagated to remote sites with decreased frequency which reduces the number of consistency messages. These two dimensions of consistency are independent. For example sequential consistency provides strong ordering but no timeliness guarantees.

In this section we describe some of the consistency protocols that we have implemented in the caching framework. The first one is an invalidation based protocol $\left(\mathrm{SC}_{i n v}\right)$ and the second one, $\mathrm{LC}_{\text {set }}$, is the local consistency (LC) protocol which is based on invalidation sets. The two protocols present different approaches for maintaining cache consistency. $\mathrm{SC}_{i n v}$ is an example of a strong consistency protocol that provides a unique ordering for all the writes in the system while providing immediate timeliness for the reads. It is used widely in shared memory systems $[8,16]$ as well as in file systems $[9,19,22] . \mathrm{LC}_{\text {set }}$ allows the timeliness threshold to be varied, but orders writes as in $\mathrm{SC}_{i n v}$. By changing the timeliness threshold for reads, $\mathrm{LC}_{\text {set }}$ allows the consistency overhead of cached objects to be varied.

Because our framework works at the object level, for the following discussion we consider method invocations on cached objects as writes or reads, depending 
on whether the method modifies the object or not. We now proceed to describe the details of the $\mathrm{SC}_{i n v}$ and the $\mathrm{LC}_{\text {set }}$ protocols.

\subsection{Server Based Invalidation Protocol $\left(\mathrm{SC}_{i n v}\right)$}

The $\mathrm{SC}_{\text {inv }}$ protocol allows either a single writer or multiple readers at a given time. We refer to the client that caches an object copy in exclusive mode as its owner. The algorithm for this protocol is presented in the Fig. 4.

When a client $\mathrm{P}_{i}$ attempts to read an object copy and experiences a readmiss, it communicates with the server. If no other client caches the object copy in exclusive mode, then the server returns its copy to the client and adds the client to its reader set. Otherwise, the server downgrades the owner's copy to read-only mode and provides $\mathrm{P}_{i}$ the latest copy from the owner. For a client $\mathrm{P}_{i}$ to perform a write operation on an object $x$, it needs to cache it in an exclusive mode. If $x$ 's copy has not been already cached or, if a copy has been cached in the shared mode, $\mathrm{P}_{i}$ experiences a write-miss, ${ }^{1}$ forcing it to communicate with the server. The server returns its copy of $x$, immediately, if the object is currently not cached by other nodes. If $x$ 's copies exist at other nodes in shared mode, then the server invalidates all such copies, and returns $x$ to client $\mathrm{P}_{i}$ in an exclusive mode. If another node happens to cache $x$ in an exclusive mode, then the server gets the recent state from that node, invalidates that copy and finally sends the most recent copy to the requester. Although this protocol orders all writes and provides immediate timeliness, its scalability, however, is limited because of the high communication costs of synchronous invalidations for update operations. The protocol presented next attempts to alleviate some of these problems.

\subsection{Invalidation-Set Protocol $\left(\mathrm{LC}_{s e t}\right)$}

The basic $\mathrm{LC}_{\text {set }}$ protocol was first presented in [1] but we have improved it in a number of ways. For example, we explore the timeliness aspect of consistency in $\mathrm{LC}_{\text {set }}$, which was not explored in earlier work. Similar to $\mathrm{SC}_{i n v}$, LC based protocols also assume a single writer for an object at a time, but there are some important differences between the invalidation protocol and those based on LC.

The $\mathrm{LC}_{\text {set }}$ protocol permits control over the timeliness aspect of the consistency of cached objects. The protocol orders all writes that are executed on a group of related objects. However, it allows a writer to update the object state while other clients are accessing the older state of the object in read only mode. The writes are not immediately propagated to all the remote sites that are caching a copy of the object. This delay may result in the reads at the remote sites returning older values of the object state. The protocol guarantees that the reads will never return a value for the object that is older than the timeliness

1 There are two cases: Write-miss and Write-fault. Write-fault occurs when the client does not have a locally cached copy of the object and a write-miss occurs when the local copy is in shared mode and needs to be upgraded. The consistency actions are similar in the two cases and we do not consider them separately. 

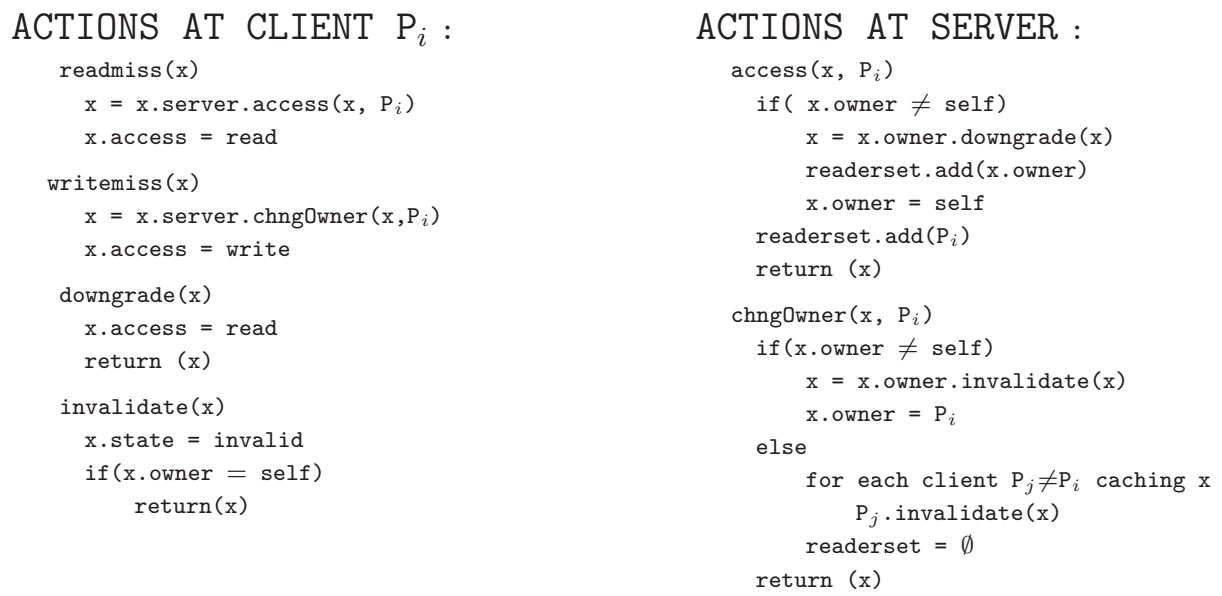

Fig. 4. Central Server Invalidation protocol $\left(\mathrm{SC}_{i n v}\right)$

threshold specified for the protocol. The client accesses that return older cached copies are serialized before the writes that create the new values. An LC based protocol orders all accesses to related objects by introducing new object copies into the node's cache in a systematic manner. At the time a new object copy is added to a node cache, the node performs local consistency actions to ensure that currently stored copies of shared objects are valid with respect to the information received with the newly fetched object. Such consistency actions are carried out based on meta-data received from the server and require no communication.

The server maintains meta-data about updates to objects and sends it to a client whenever the client communicates with it. There are two cases in which it becomes necessary for the client to communicate with the server. First, all misses require communication with the server. The server does not send messages to invalidate other copies in the system when a write-miss request is received. Instead, information about the object that needs to be invalidated is recorded in an invalset (for invalidation set). Because invalset stores the identities of those objects which have been updated but the update related consistency messages have not been propagated to the caching clients, invalset continuously evolves as objects are updated and communication takes place between the nodes. We can associate a time with each member of invalset. This can be used to determine when updates to certain objects were done. The functions update and merge in Fig. 5 are used to update an invalset. update adds an object to invalset when the object is updated and also includes the time at which this change to the invalset is made (if the object is already in invalset, then only the time is changed). The merge function merges two invalidation sets by keeping the information about the most recent update to each object listed in the two sets. The newerthan function in the algorithm uses timing information to order updates to an object. The function newWrite is called during each 
and allows it to continue as the owner. Also, the invalset is returned to the client as in the case of write misses. When a client receives an invalset from the server, it invalidates the cached copies of objects listed in the invalset that have been overwritten. Thus, object copies at a client are invalidated only when the client communicates with the server and not when the object is written.

There are two different ways in which the server can maintain the invalset. It can either maintain the set on a per-client basis or can have a single invalset for all the clients. We chose the latter approach in our protocol to improve scalability and to reduce the computation costs associated with updating each client's invalset, when an object is updated. The invalset can be viewed as a table of records indexed by the object-id. Each record in the table is the tuple $\{$ modify-bit, epoch $\}$. A value of 1 for the modify-bit indicates that the object is currently cached in an exclusive mode by a client. If the invalset does not contain an entry for an object, then it means that either the object has not been cached by any client or is being cached in read-only mode by all the clients. The epoch number can be considered as a generation identifier indicating how old the object is. The server increments this epoch number whenever it receives a new copy of the object from its current owner. The clients locally maintain an epoch vector for all the objects they cache. Whenever a client receives the invalidation set, it compares the epoch received for each object with its local epoch number and it invalidates the cached copy only if the new epoch number is greater than the local one. This ensures that the client does not invalidate an object if it has the latest copy of the object.

An example of how the invalset is used by $\mathrm{LC}_{\text {set }}$ to ensure ordering is shown in Fig. 6. There are three clients $C_{1}, C_{2}$ and $C_{3}$ and the server $S$. The application has three shared objects, $O_{1}, O_{2}$ and $O_{3}$ that get instantiated at the server at the start. The figure also shows the sequence of operations executed at the clients. The table in Fig. 6 gives a possible global serialization order of the operations as provided by the $\mathrm{LC}_{\text {set }}$ protocol, the consistency action description, the state of the cached objects at the clients before and after the execution of consistency actions, and the value of the epoch numbers in the invalset at the clients after the completion of consistency actions. The clients first populate their caches with the initial state of $\mathrm{O}_{1}, \mathrm{O}_{2}$ and $\mathrm{O}_{3}$ which are respectively $v_{1}, u_{1}$, and $z_{1}$. As seen from the figure, the operation $c_{1}: w\left(O_{1}\right) v_{2}$ at client $\mathrm{C}_{1}$ triggers the consistency action $C_{1} \rightarrow S$ (from $C_{1}$ to $S$ ), which grants the exclusive ownership of $\mathrm{O}_{1}$ to $\mathrm{C}_{1}$. It also changes the invalset at $\mathrm{C}_{1}$ and $\mathrm{S}$ to 100 , indicating that $\mathrm{O}_{1}$ has been modified. During the write operation $c_{2}: w\left(O_{2}\right) u_{2}$ at $\mathrm{C}_{2}$, the consistency operation $C_{2} \rightarrow S$, modifies the invalset at $\mathrm{C}_{2}$ to [110]. Since the epoch of $\mathrm{O}_{1}$ in the received invalset is higher than the locally stored epoch, $\mathrm{C}_{2}$ invalidates its local copy of $\mathrm{O}_{1}$. During the next operation $c_{2}: r\left(O_{1}\right) v_{2}, \mathrm{C}_{2}$ experiences a read miss. The consistency action $C_{2} \rightarrow S \rightarrow C_{1}$ brings the new state of $\mathrm{O}_{1}$ to $\mathrm{C}_{2}$ from $\mathrm{C}_{1}$. Also during this transfer, a new invalidation set 110 is propagated to $\mathrm{C}_{1}$. Again $\mathrm{C}_{1}$ invalidates $\mathrm{O}_{2}$ because of the higher epoch number received. When $\mathrm{C}_{3}$ writefaults during the operation $c_{3}: w\left(\mathrm{O}_{3}\right) z_{2}$, S sends the invalset [110] to $\mathrm{C}_{3}$. $\mathrm{C}_{3}$ invalidates the local copies of $\mathrm{O}_{1}$ and $\mathrm{O}_{2}$ because their new epoch numbers 
are greater than the local epoch values. Subsequent read operations on them experience read misses and the new states of $\mathrm{O}_{1}$ and $\mathrm{O}_{2}$ are brought in from $\mathrm{C}_{1}$ and $\mathrm{C}_{2}$.

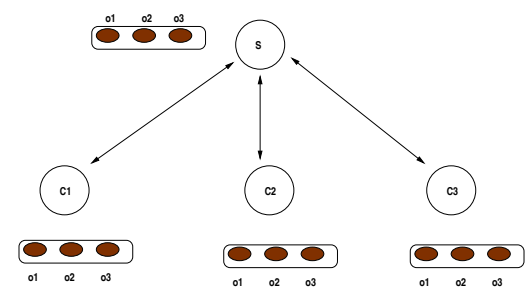

c1:r(01)v1, r(02)u1, r(03)z1, w(01)v2, r(02)u1, r(03)z1 c2:r(01)v1, r(02)u1, r(03)z1, w(02)u2, r(01)v2, r(03) z1

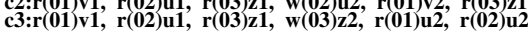

\begin{tabular}{|c|c|c|c|c|c|c|c|c|c|c|c|c|c|}
\hline \multirow{2}{*}{$\begin{array}{l}\text { Global Order } \\
\text { provided by } L_{\text {set }}\end{array}$} & \multicolumn{4}{|c|}{$\begin{array}{c}\text { State } \\
\text { Before Action }\end{array}$} & \multirow[t]{2}{*}{$\begin{array}{c}\text { Consistency } \\
\text { Actions }\end{array}$} & \multicolumn{4}{|c|}{$\begin{array}{c}\mathrm{New} \\
\text { invalidation-set at }\end{array}$} & \multicolumn{4}{|c|}{$\begin{array}{c}\text { State } \\
\text { After Action }\end{array}$} \\
\hline & At & 01 & 02 & 03 & & C1 & C2 & C3 & $S$ & At & 01 & \begin{tabular}{|l|}
02 \\
\end{tabular} & 03 \\
\hline$c 1: r(01) v 1$ & C1 & u & $\mathrm{u}$ & U & $C 1->S$ & \begin{tabular}{|l|l|l|}
0 & 0 & 0 \\
\end{tabular} & null & null & \begin{tabular}{|l|l|l|}
0 & 0 & 0 \\
\end{tabular} & C1 & $\mathrm{s}$ & $\mathrm{u}$ & $u$ \\
\hline$c 1: r(02) u 1$ & C1 & $\mathrm{s}$ & $\mathrm{u}$ & u & C1 $1>\mathrm{S}$ & \begin{tabular}{|l|l|l|}
0 & 0 & 0 \\
\end{tabular} & null & null & \begin{tabular}{|l|l|l|}
0 & 0 & 0 \\
\end{tabular} & C1 & $\mathrm{s}$ & $\mathrm{s}$ & u \\
\hline c1:r(03)z1 & C1 & $\mathrm{s}$ & $\mathrm{s}$ & u & C1->S & \begin{tabular}{|l|l|l|}
0 & 0 & 0 \\
\end{tabular} & null & null & \begin{tabular}{|l|l|l|}
0 & 0 & 0 \\
\end{tabular} & C1 & $\mathrm{s}$ & $\mathrm{s}$ & $\mathrm{s}$ \\
\hline c2:r(01)v1 & C2 & u & $u$ & U & C2->S & \begin{tabular}{|l|l|l|}
0 & 0 & 0 \\
\end{tabular} & \begin{tabular}{|l|l|l|}
0 & 0 & 0 \\
\end{tabular} & null & \begin{tabular}{|l|l|l|}
0 & 0 & 0 \\
\end{tabular} & C2 & $\mathrm{s}$ & u & u \\
\hline c2:r(02)u1 & C2 & $\mathrm{s}$ & U & U & C2 $\rightarrow S$ & \begin{tabular}{|l|l|l|}
0 & 0 & 0 \\
\end{tabular} & \begin{tabular}{|l|l|l|}
0 & 0 & 0 \\
\end{tabular} & null & \begin{tabular}{|l|l|l|}
0 & 0 & 0 \\
\end{tabular} & C2 & $\mathrm{s}$ & $\mathrm{s}$ & U \\
\hline c2:r(03)z1 & C2 & S & $\mathrm{s}$ & U & C2 $\rightarrow S$ & \begin{tabular}{|l|l|l|}
0 & 0 & 0 \\
\end{tabular} & \begin{tabular}{|l|l|l|}
0 & 0 & 0 \\
\end{tabular} & null & \begin{tabular}{|l|l|l|}
0 & 0 & 0 \\
\end{tabular} & C2 & $\mathrm{s}$ & $\mathrm{s}$ & $\mathrm{s}$ \\
\hline c3:r(01)v1 & C3 & $\mathrm{u}$ & u & u & C3->S & \begin{tabular}{|l|l|l|}
0 & 0 & 0 \\
\end{tabular} & \begin{tabular}{|l|l|l|}
0 & 0 & 0 \\
\end{tabular} & \begin{tabular}{|l|l|l|}
0 & 0 & 0 \\
\end{tabular} & \begin{tabular}{|l|l|l|}
0 & 0 & 0 \\
\end{tabular} & C3 & $\mathrm{s}$ & u & U \\
\hline c3:r(02)u1 & C3 & $\mathrm{s}$ & U & u & C3->S & \begin{tabular}{|l|l|l|}
0 & 0 & 0 \\
\end{tabular} & \begin{tabular}{|l|l|l|}
0 & 0 & 0 \\
\end{tabular} & \begin{tabular}{|l|l|l|}
0 & 0 & 0 \\
\end{tabular} & \begin{tabular}{|l|l|l|}
0 & 0 & 0 \\
\end{tabular} & C3 & s & $\mathrm{s}$ & U \\
\hline c3:r(03)z1 & C3 & s s & $\mathrm{s}$ & u & C3 $\rightarrow S$ & \begin{tabular}{|l|l|l|}
0 & 0 & 0 \\
\end{tabular} & \begin{tabular}{|l|l|l|}
0 & 0 & 0 \\
\end{tabular} & \begin{tabular}{|l|l|l|}
0 & 0 & 0 \\
\end{tabular} & \begin{tabular}{|l|l|l|}
0 & 0 & 0 \\
\end{tabular} & C3 & $\mathrm{s}$ & $s$ & $\mathrm{~s}$ \\
\hline$c 1: w(01) v 2$ & C1 & $\mathrm{s}$ & $\mathrm{s}$ & $\mathrm{s}$ & C1->S & 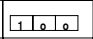 & \begin{tabular}{|l|l|l|}
0 & 0 & 0 \\
\end{tabular} & \begin{tabular}{|l|l|l|}
0 & 0 & 0 \\
\end{tabular} & \begin{tabular}{|l|l|l|}
1 & 0 & 0 \\
\end{tabular} & C1 & $x$ & $s$ & s \\
\hline$c 1: r(02) u 1$ & C1 & $x$ & $s$ & $\mathrm{~s}$ & None & \begin{tabular}{|l|l|l|} 
& 0 & 0 \\
\end{tabular} & \begin{tabular}{|l|l|l|}
0 & 0 & 0 \\
\end{tabular} & \begin{tabular}{|l|l|l|}
0 & 0 & 0 \\
\end{tabular} & \begin{tabular}{|l|l|l|}
1 & 0 & 0 \\
\end{tabular} & C1 & $\mathrm{x}$ & $\mathrm{s}$ & $\mathrm{s}$ \\
\hline c1:r(03)z1 & C1 & $\mathrm{x}$ & $\mathrm{s}$ & $s$ & None & \begin{tabular}{|l|l|l|} 
& 0 & 0 \\
\end{tabular} & \begin{tabular}{|l|l|l|}
0 & 0 & 0 \\
\end{tabular} & \begin{tabular}{|l|l|l|}
0 & 0 & 0 \\
\end{tabular} & \begin{tabular}{|l|l|l|}
1 & 0 & 0 \\
\end{tabular} & C1 & $\mathrm{x}$ & $\mathrm{s}$ & $\mathrm{s}$ \\
\hline $\mathrm{c} 2: \mathrm{w}(02) \mathrm{u} 2$ & C2 & $\mathrm{s}$ & $\mathrm{s}$ & $\mathrm{s}$ & C2 $\rightarrow S$ & \begin{tabular}{|l|l|l|} 
& 0 & 0 \\
\end{tabular} & \begin{tabular}{|l|l|l|} 
& 1 & 0 \\
\end{tabular} & \begin{tabular}{|l|l|l|}
0 & 0 & 0 \\
\end{tabular} & \begin{tabular}{|l|l|l|} 
& 1 & 0 \\
\end{tabular} & C2 & $\mathrm{I}$ & $x$ & $\mathrm{~s}$ \\
\hline c2:r(01)v2 & C2 & 1 & $x$ & $\mathrm{~s}$ & $\mathrm{C} 2->\mathrm{S}->\mathrm{C} 1$ & \begin{tabular}{|l|l|l|} 
& 1 & 0 \\
\end{tabular} & \begin{tabular}{|l|l|l|}
1 & 1 & 0 \\
\end{tabular} & \begin{tabular}{|l|l|l|}
0 & 0 & 0 \\
\end{tabular} & \begin{tabular}{|l|l|l|}
1 & 1 & 0 \\
\end{tabular} & C2 & $\mathrm{s}$ & $x$ & $\mathrm{~s}$ \\
\hline c2:r(03)z1 & C2 & $\mathrm{s}$ & $x$ & $\mathrm{~s}$ & None & \begin{tabular}{|l|l|l|}
1 & 1 & 0 \\
\end{tabular} & \begin{tabular}{|l|l|l|}
1 & 1 & 0 \\
\end{tabular} & \begin{tabular}{|l|l|l|}
0 & 0 & 0 \\
\end{tabular} & \begin{tabular}{|l|l|l|}
1 & 1 & 0 \\
\end{tabular} & C2 & $\mathrm{s}$ & $x$ & $\mathrm{~s}$ \\
\hline c3:w(03)z2 & C3 & $\mathrm{s}$ & $s$ & $s$ & C3->S & \begin{tabular}{|l|l|l|}
1 & 1 & 0 \\
\end{tabular} & \begin{tabular}{|l|l|l|}
1 & 1 & 0 \\
\end{tabular} & \begin{tabular}{|l|l|l|}
1 & 1 & 1 \\
\end{tabular} & \begin{tabular}{|l|l|l|}
1 & 1 & 1 \\
\end{tabular} & C3 & 1 & 1 & $x$ \\
\hline c3:r(01)v2 & C3 & 1 & 1 & $x$ & C3->S->C1 & \begin{tabular}{|l|l|l|}
1 & 1 & 1 \\
\end{tabular} & \begin{tabular}{|l|l|l|}
1 & 1 & 0 \\
\end{tabular} & \begin{tabular}{|l|l|l|}
1 & 1 & 1 \\
\end{tabular} & \begin{tabular}{|l|l|l|}
1 & 1 & 1 \\
\end{tabular} & C3 & $\mathrm{s}$ & 1 & $x$ \\
\hline c3:r(02)u2 & C3 & s & 1 & $x$ & $\mathrm{C} 3->\mathrm{S}->\mathrm{C} 2$ & \begin{tabular}{|l|l|l|}
1 & 1 & 1 \\
\end{tabular} & \begin{tabular}{|l|l|l|}
1 & 1 & 1 \\
\end{tabular} & \begin{tabular}{|l|l|l|}
1 & 1 & 1 \\
\end{tabular} & \begin{tabular}{|l|l|l|}
1 & 1 & 1 \\
\end{tabular} & C3 & $\mathrm{s}$ & $\mathrm{s}$ & $x$ \\
\hline
\end{tabular}

Fig. 6. An example of Invalidation-set protocol.The figure shows the new invalidation-sets at the three clients $C_{1}, C_{2}$ and $C_{3}$ and the server $S$ when the shared objects $\mathrm{O}_{1}, \mathrm{O}_{2}$ and $\mathrm{O}_{3}$ are read or written

Clients that do not communicate with the server, will continue to read the copies cached by them. To guarantee that the cached copy of an object does not remain stale for more than the specified timeliness threshold, periodic communication between the client and the server is necessary. This could be ensured in the following two ways.

- The server could maintain a global time clock and after every refresh-period force the clients to invalidate the locally cached copies, by sending them the latest invalset. This is a push based approach. 
- In a pull based approach, each client can locally timeout when the communication has not taken place with the server for a certain period of time. The client can then locally invalidate the objects with expired timeliness so that the next invocation on them will result in the client communicating with the server and getting a more recent invalset.

We chose the second option to provide clients control over when to fetch the object. Also, this way different clients can choose different timeliness thresholds for their cached copies based on their needs and bandwidth availability. In our implementation, whenever an object state is renewed at the client, it also gets timestamped. The function timeliness Check called during each invocation checks if the time since the last communication with the server for that particular object exceeds its timeliness bound. If so, then the object is marked invalid. This will trigger a readmiss or a writemiss, forcing the client to communicate with the server to retrieve the latest copy. The client will also receive the invalset for the entire set of cached objects.

\subsection{Implementation of Consistency Protocols}

This section describes some implementation specific issues of the caching framework. In particular, the programming interface to the caching system. The consistency protocols are all implemented as Java classes and have a generic parent class defining methods that are common to all the protocols. The parent class also implements interfaces through which the consistency protocol objects may be accessed from outside. The actual definitions of the methods in these interfaces may be delegated to the consistency policy class implementing a particular consistency protocol. The details are as follows.

Consistency protocols implemented by the consistency objects on both the client and server sides extend the ConsistencyModel class. They also implement the ConsistencyScheme interface. The ConsistencyModel Class has a generic set of routines that are suitable for any consistency protocol. The ConsistencyScheme interface defines methods, through which the consistency object is accessed. In addition, the client side consistency object implements the ConsistencyScheme_Client interface and the server side object implements the ConsistencyScheme_Server interface. These interfaces contain methods specific to the client side and the server side of the caching framework.

The ConsistencyModel is an abstract class. It contains definitions for the methods that are common to all the consistency protocols and abstract methods which are more protocol specific. Some of these methods are the following.

- setProtocol - It is used to set the consistency policy of the caching framework.

- fetchLatestCopy - It ensures that the reference to the cached copy is in a valid state. It is an abstract method, whose definition is delegated to a particular consistency protocol object.

- takeActions - It is invoked when a request for a consistency action is received from other consistency objects at server or at other clients. The actual definition of the method is delegated to the protocol object. 
The ConsistencyScheme interface provides access points through which the caching framework can be accessed from the outside (e.g. delegate). It declares the following two functions:

- guard- It is invoked before the actual method invocation. This method locks the object so that no consistency related actions are performed on the object during the execution of the method.

- relax - It is invoked after the execution of the method is completed. It releases the lock acquired during the guard method .

We chose an approach in which the framework provides synchronized access to object state rather than delegating it to the object implementor. By allowing the framework to control synchronization, we can allow invocations that only read the object state to execute simultaneously at a node, increasing parallelism.

The ConsistencyScheme_Client interface declares the method cacheObject, which is called once when the object is cached for the first time at the client node. The method adds the object to the set of objects cached at the client. The ConsistencyScheme_Server interface declares the cacheObject method which is called once when an object is exported at the server node.

\section{Performance}

So far we have discussed the architecture of the caching system and the consistency protocols that have been implemented to ensure that operations are executed with consistent object state. The goal of this section is to experimentally evaluate the performance of the caching system to quantify the improvements that can be achieved by caching, and the impact of the consistency protocols on the performance of caching. We first measure the costs associated with the basic operations in the system such as the overhead imposed by the caching framework to execute invocations that read or write the state of the invoked object. This is followed by a more detailed evaluation of the system with a synthetic workload derived from attributes of an interactive distributed application.

The experiments were conducted on a cluster of $248 \mathrm{MHz}$ Sparc Ultra-30's connected by a $100 \mathrm{Mb}$ Ethernet. The machines were all equipped with 128 MBytes of memory. The Java virtual machine used was Java2 from Javasoft and we used it with the just-in-time (JIT) option enabled. There were no other applications running on the machines when the experiments were conducted and hence the numbers generated were repetitive. We ran each of the experiments three times and the numbers presented here are averaged across multiple runs and over multiple clients. It was difficult to generate numbers that were repetitive for a wide-area configuration. This was primarily due to our lack of control on the network. Because of this reason, we are only presenting the measurements for the local-area environment in this paper. In the future, we plan to repeat these with widely distributed sites connected by the Internet, possibly using an Internet emulation testbed. 
In our experiments clients invoked an object $O$ implemented by the server. The definition of $\mathrm{O}$ has two member functions: read(), and write(). The read method has a null body while the write method increments the state of a shared counter. Since little time is spent in the execution of the methods, the average invocation time obtained in the experiments is a direct measure of the communication and computation costs associated with the caching framework.

\subsection{Basic Performance of Caching}

Object caching enables a remote invocation to be completed locally when the cached object is in a valid state and in proper mode. If the cached copy is not consistent, the invocation will result in the client communicating with the server to fetch the current copy which could generate one or more messages between the server and clients. To measure the cost of completing a remote invocation in different situations, we decided to measure the cost of invocations in the following cases.

- The object is invoked remotely at the server without caching it locally. Thus, this will be the base case where the existing Java RMI framework is used to make the invocation at the remote server.

- The object is cached locally and is in a valid state and correct mode. This invocation is local and will not result in any communication with the server. This is the best case for caching.

- The object is locally cached but is not in a valid state, but a valid copy can be found at the server. This will result in the client communicating with the server, fetching the new copy from it.

- The object is locally cached and is not in a valid state. A valid copy can be found at some other remote client $C_{n}$. This will result in the client communicating with the server followed by the server communicating with $\mathrm{C}_{n}$, which will return a copy via the server to the requesting client.

- The object is cached locally in shared mode and the invocation needs an exclusive copy. The client has to communicate with the server, and the server with other remote clients depending on the consistency protocol.

Table 1. Comparison of the time per invocation in milliseconds averaged over 10,000 invocations. The invocations were executed at the server and with locally cached copies. The size of the object was 1024 bytes and a group of 8 related objects were used

\begin{tabular}{ll}
\hline Invocation Execution & Invocation Time in milliseconds \\
\hline At remote server & 1.244 \\
At locally cached copy & 0.025 \\
\hline
\end{tabular}


Table 2. Comparison of time per invocation in milliseconds averaged over 10,000 invocations. The invocations were executed at the cached object with a writemiss resulting in the new state being fetched from the server. The size of the object was 1024 bytes and the group had 8 objects. The Reader-set size corresponds to the number of other clients in the system that had the object cached in the shared state

\begin{tabular}{cccccc}
\hline Consistency actions for Protocol & \multicolumn{4}{c}{ Number of Clients } \\
Invocation Execution & & \multicolumn{4}{c}{ caching the object } \\
& & 0 & 2 & 4 & 8 \\
\hline With write-miss and & $\mathrm{SC}_{\text {inv }}$ & 3.286 & 5.631 & 8.124 & 13.532 \\
copy fetched from server & $\mathrm{LC}_{\text {set }}$ & 3.575 & 3.575 & 3.575 & 3.575 \\
\hline
\end{tabular}

Table 1 compares the costs of executing an invocation at the server and on the locally cached copy. Eight objects, each of size 1024 bytes were used in the evaluation. The average cost of a local invocation is about 25 microseconds, while it takes 1.244 milliseconds for the remote execution to take place at the server. Thus, there is a fifty fold improvement in performance if the execution can be done locally. Table 2 compares the costs of similar actions, for cached invocations when $\mathrm{SC}_{i n v}$ and the $\mathrm{LC}_{\text {set }}$ protocols are employed for consistency maintainence. For a reader to fetch a new copy from a writer via the server, it costs about 3.575 milliseconds when using the $\mathrm{LC}_{\text {set }}$ protocol. If every two out of three invocations can be executed locally, then the total time spent on the invocations is about 3.625 milliseconds, while the three remote invocations at the server require 3.732 milliseconds. So for a hit-ratio greater than $67 \%$, the performance of $\mathrm{LC}_{\text {set }}$ is better than executing the invocations at the server.

Table 2 also shows an interesting difference in the two consistency protocols. As the size of the reader-set increases, there is an increase in the invocation time for $\mathrm{SC}_{i n v}$, while it does not change for the $\mathrm{LC}_{\text {set }}$ protocol. This is because $\mathrm{SC}_{\text {inv }}$ allows either one exclusive owner or multiple shared readers to co-exist. Therefore, before it can grant access to an exclusive copy for a write-miss, it has to invalidate all the clients of the reader-set. The $\mathrm{LC}_{\text {set }}$ allows one exclusive writer and multiple shared readers to co-exist at the same time. A request for write-miss does not result in any immediate invalidation messages from the server to the reader clients. Hence the invocation cost does not vary with the number of clients caching the object in the shared mode.

Table 3 compares the invocation cost for the $\mathrm{LC}_{\text {set }}$ protocol for objects of different sizes ( 8 bytes and 1024 bytes). There is a $20 \%$ increase in the invocation time when the size of the invalidation-set is increased from 1 to 16 objects. This can be attributed to the additional computation and communication costs involved in marshalling and unmarshalling larger invalidation-set objects. 
Table 3. Time per invocation in milliseconds averaged over 10,000 invocations for the $\mathrm{LC}_{\text {set }}$ protocol

\begin{tabular}{lllllll}
\hline Consistency actions for & \multicolumn{5}{c}{ Object Size Invalidation-Set Size in objects } \\
Invocation Execution & in bytes & 1 & 2 & 4 & 8 & 16 \\
\hline With read-miss and & 8 & 2.902 & 2.961 & 2.900 & 3.112 & 3.525 \\
copy fetched from server & 1024 & 3.296 & 3.309 & 3.401 & 3.575 & 3.955 \\
With read-miss and copy fetched 8 & 5.477 & 5.482 & 5.488 & 5.830 & 6.421 \\
from remote client via the server & 1024 & 5.859 & 5.952 & 5.959 & 6.328 & 6.912 \\
\hline
\end{tabular}

\subsection{Workloads}

Workload Modeling The cost of basic operations in the caching framework clearly reveals that significant improvements can be achieved if invocations are executed with cached copies. However, locality of access, which determines when the cached copies can be used, depends on the behavior of the applications. Hence, it is desirable to evaluate the system using actual distributed applications. Unfortunately currently available traces are mostly from the distributed file system $[5,9,10,19,22]$ and the world-wide web $[20,21]$ domains. The read/write sharing patterns for these are more coarse grained and often there is a single writer for a given object. Hence we chose not to use these traces to evaluate our system. Since interactive applications can involve actual users and their behavior can depend on response time for their actions, it is difficult to create real traces [4]. We decided to use synthetically generated workloads based on important parameters of interactive applications like Aquamoose described earlier. Our synthetic workload can be described by the following parameters. The values associated with the parameters below were those used in generating the traces.

Number of Objects: There are $N$ shared objects $O_{1}, O_{2}, \ldots, O_{n}$ each of size $S_{1}, S_{2}, \ldots, S_{n}$ bytes that are governed by the consistency protocol. They are all instantiated at the same server. In our experiment we assigned a value of 32 to $\mathrm{N}$ and all of $\mathrm{S}_{1}, \mathrm{~S}_{2}, \ldots, \mathrm{S}_{n}$ were assigned a value of 64 .

Number of Clients: There are $C$ clients that can make invocations on the objects. We assigned a value of 8 to $\mathrm{C}$.

Number of Invocations per Client: Each client makes $K$ invocations. K was chosen to be 10,000.

Read Frequency: Assuming that interactive applications are visual and require frequent screen updates, we generated read request to the objects once in every 30 milliseconds.

Write Frequency: The writes in these applications may be because of user actions or because of movement of autonomous entities (e.g., movement of fish in a predetermined trajectory). We also assumed that a user does not recognize events happening in a time period less than $100 \mathrm{~ms}$. So the lower limit for the 
time between writes is $100 \mathrm{~ms}$ (for autonomous entity movement) and the higher limit was fixed at 3 seconds (for user actions). The writes were generated at random with the above mentioned higher and lower time bounds.

Ownership: The ownership was assumed to be static for this trace. This is a reasonable assumption because in many distributed applications like the virtual world, the changes to object state are made only by the users who created them.

We use a trace generated based on the above parameters to evaluate the two protocols.

Performance Evaluation The performance metrics that are of interest are the average invocation time and the number of cache misses and server requests. The experiments were conducted in the same lab environment in which the basic benchmark tests were done.

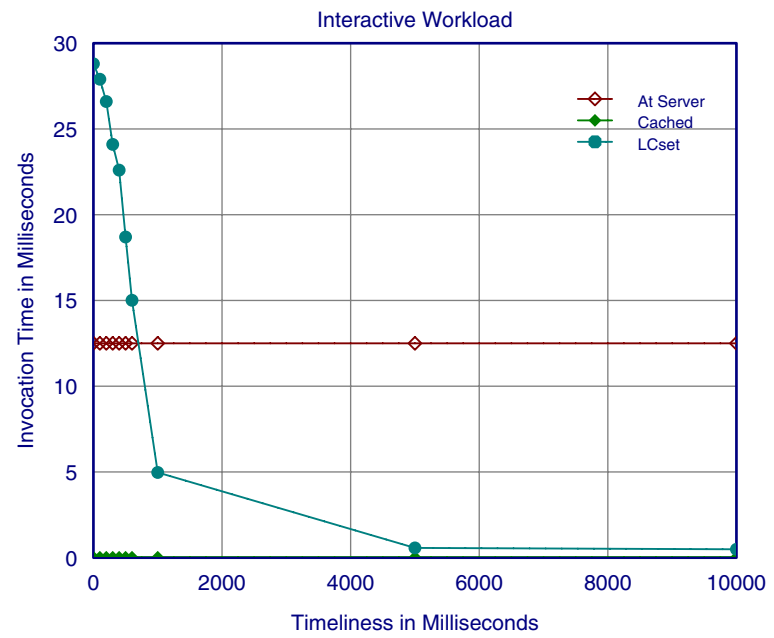

Fig. 7. The invocation time averaged over 10000 invocations with 8 clients and 32 objects. The invocation time for the method execution at the server is 12.52 milliseconds while the invocation time for the method execution on the local copy is 25 microseconds

Fig. 7 shows the average execution times for invocations at the server, locally cached invocation and local invocation with the $\mathrm{LC}_{\text {set }}$ protocol, for different values of the timeliness threshold. The average invocation time for execution at the server is 12.52 milliseconds. This is different from the micro-benchmark results and can be explained by the increase in server load because of 8 clients operating simultaneously while the average invocation time for a locally cached copy is only 25 microseconds. The invocation time for the $\mathrm{LC}_{\text {set }}$ protocol is an exponentially decreasing curve. It is about 28.84 milliseconds when the timeliness 
threshold is 0 and reduces to about 746 microseconds as the timeliness threshold value is increased to 5 seconds. This can be explained from the way the protocol works. The clients invalidate their local copies whenever they receive an invalidation-set from the server or whenever the timeliness threshold expires. When the timeliness threshold is set to 0 , the object copy expires as soon as it is locally cached. Hence all the invocations find the copy invalid, leading to consistency actions. This accounts for higher method execution times. However, when the timeliness threshold is increased, such invalidation and the resulting communication decreases The system initially invalidates the objects when it receives the invalidation-sets. As the steady state is reached, the cache gets populated with objects in valid state. Thus, invalidation frequency due to the receipt of invalidation-set decreases and beyond a point, the invalidations are only due to the expiration of the timeliness threshold. This explains why the number of cache-misses decrease as the timeliness threshold is increased, leading to much smaller execution times for the invocations.

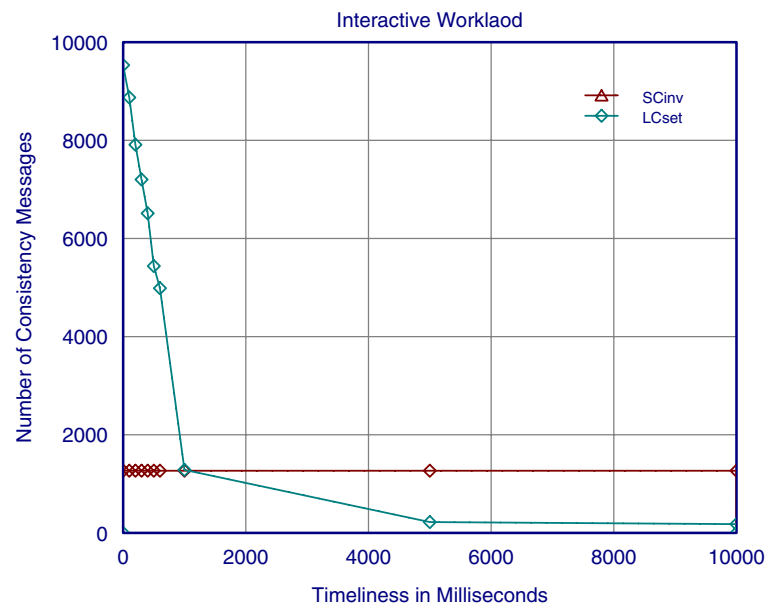

Fig. 8. The number of cache misses for 10000 invocations with 8 clients and 32 objects. The cache misses generated for the $\mathrm{SC}_{i n v}$ protocol is 1267

Fig. 8 shows the number of cache misses for the protocols $\mathrm{SC}_{i n v}$ and $\mathrm{LC}_{\text {set }}$. The number of consistency messages generated by the $\mathrm{SC}_{i n v}$ protocol does not depend on the timeliness value. While for the $\mathrm{LC}_{\text {set }}$ protocol, it is an exponentially decreasing curve. At a timeliness threshold of 0 , every invocation other than writes (because of the exclusive ownership assumption) will result in a cache-miss for reasons explained earlier. This is why we experience 9532 cache misses for the 10000 invocations executed during the experiment. As timeliness threshold is increased, a lot more invocations are completed with the cached object and hence the cache misses decrease. 


\section{Related Work}

Rich interactive distributed applications like Spline[3], Aquamoose [6] and other virtual reality (VR) applications have been developed in the recent past which could involve large numbers of geographically distant users interacting in real time. In addition to interacting with each other, users also interact with computer simulations which range from the very simple (e.g., a revolving door) to the very complex (e.g., a human-like robot). These applications also allow users to make temporary and permanent modifications and extensions to the environment while they are running, so that the content of an environment can dynamically change. For reasons of efficiency, consistency and scalability, these applications are built with abstractions provided by a distributed communication infrastructure. Some examples of such infrastructures include the MR toolkit [26] and the $d V S$ system [12]. They assume a client-server topology, where a client is represented by a collection of processes. It uses message passing paradigm to disseminate information between the participants. Any change in the state at a client is propagated by the client's network process to every other client's network process. DIVE [15] uses shared memory paradigm and uses object abstractions for shared state. An ownership protocol and multicast mechanisms are used to maintain the consistency of the state. SIMNET [7] and NPSNET [18] are used to develop military battlefield simulations. They use a combination of shared memory and message passing mechanisms. They use best-effort broadcast or multicast to communicate user actions to remote sites. Scalability is achieved by locally maintaining a copy of remote state and simulating remote actions through one of the allowed set of behaviors of the application. High Level Architecture (HLA) [11] is a system where messages across nodes can be ordered at the receiver using one of the following ordering types: receive order, priority order, causal order, time stamp order etc. Our distributed object caching system also provides a platform that can be used by the applications mentioned earlier. But it differs from them by allowing clients to specify their timeliness constraints through a high level QoS interface. By separating out the QoS interface from the functional part of the application, our system can execute the same application program at different locations, but in different QoS domains. For example, different clients can define different timeliness thresholds based on their need and resources available to them. At the same time, the system can provide consistency via its consistency protocols. It also tries to optimize communication overhead based on consistency policies used. The system can adaptively change its timeliness requirements at runtime based on available resources and application needs.

Commercial ORBs like Orbix[24] from Iona and Visigenic[23] from Borland have a proxy technology that can be used to add caching to their remote object framework. These are called smart proxies in Orbix and interceptors in Visigenic. We could have used these systems but we chose $\mathrm{QuO}$ because $\mathrm{QuO}$ proxies are generated from a high level QoS description language. Thus, it was easy to add shared state QoS to the QuO framework. Other object systems such as Globe[25] have multiple physical copies of an object residing on different machines. Clients 
may contact any copy to get methods executed without knowing the internal structure and protocols used by the object implementation. This scheme allows different objects to use different algorithms for data partitioning, replication, consistency, and fault tolerance, in a way transparent to the users. We focus on object caching and the consistency aspects of the cached copies in a QoS framework.

A lot of consistency related work has been done in the areas of distributed shared memory, distributed file systems and the world-wide web. Consistency protocols for the web are described in $[14,17,27]$. Weak consistency protocols based on time to live (TTL) for timeliness are presented in [14]. Although these weaker consistency protocols provide better scalability and enhance system performance, their notions of consistency are too weak to support interactive applications. Stronger notions of consistency based on invalidation and polling for the web are presented in [17]. Different distributed file systems like XFS [9] employ caching and replication to enhance performance. The invalidation based consistency protocols used by them disallow the co-existence of readers and writers and are more expensive to implement. The $\mathrm{LC}_{\text {set }}$ protocol described in this paper provides strong consistency but allows readers and a writer to co-exist, and explicitly addresses the timeliness dimension of consistency, providing consistency vs. cost tradeoffs.

\section{Conclusions}

In this paper we have presented the architecture of an object caching system that transparently caches objects. The framework is configurable and multiple consistency protocols are used to govern the state of the cached objects based on application requirements and resource availability. The high level specification of the consistency requirements is done through the Quality Object's Quality Description Languages. We also presented the details of the caching framework for the specification and governance of the consistency QoS requirements.

We have implemented two consistency protocols, a server based strict consistency protocol, $\mathrm{SC}_{i n v}$ which provides a strict serialization order for all the reads and writes in the system and the $\mathrm{LC}_{\text {set }}$ protocol which provides a more relaxed ordering for reads depending on the value of the timeliness threshold. The $\mathrm{LC}_{\text {set }}$ protocol provides better response time for invocations, almost as small as an invocation on a local object, for interactive workloads, for a timeliness threshold value greater than 1 second. Also, the number of consistency messages needed for the $\mathrm{LC}_{\text {set }}$ protocol is much lower than the the $\mathrm{SC}_{i n v}$ protocol for higher timeliness thresholds.

In the future we would like to develop additional consistency protocols for the framework providing different levels of guarantees based on the timeliness and the ordering requirements. Also, we would like to develop synthetic workloads for interactive applications. Actual experiments and simulations driven by the workloads will allow us to perform a detailed evaluation of the protocol imple- 
mentations. We are also interested in developing some applications with which we can drive the framework, to determine the effectiveness of the system.

\section{References}

1. M. Ahamad and R. Kordale. Scalable Consistency Protocols for Distributed Services. IEEE Transcations on Parallel and Distributed Systems, 1999. 55

2. M. Ahamad, G. Neiger, J. E. Burns, P. W. Hutto, and P. Kohli. Causal Memory: Definitions, Implementation and Programming. Distributed Computing, 9:37-49, 1995. 54

3. D. W. Anderson ,J. W. Barrus, J. W. Barrus, J. W. Howard, C. Rich, C. Shen, and R. C. Waters. Building Multi-User Interactive Multimedia Environments in MERL. IEEE Multimedia, 2(4):77-82, 1995. 66

4. S. Bhola and M. Ahamad. Workload Modeling for Highly Interactive Distributed Applications. Technical Report GIT-CC-99-2, College of Computing, Georgia Institute of Technology, 1999. 64

5. M. Blaze. Caching in Large-Scale Distributed File Systems. PhD thesis, Princeton, 1992. 64

6. A. Bruckman. Community Support for Constructionist Learning. In Proc. of the 7th ACM Conference on Computer Supported Cooperative Work, 1998. 47, 66

7. J. Calvin, A. Dickens, B. Gaines, P. Metzger, D. Miller, and D. Owen. The SIMNET Virtual World Architecture. In Proceedings of the IEEE VRAIS, pages 450-455, 1993. 67

8. J. Carter, J. Bennett, and W. Zwaenepoel. Implementation and Performance of Munin. In Proceedings of the Thirteenth Symposium on Operating Systems Principles (SOSP), pages 152-164, October 1991. 54

9. M. D. Dahlin, R. Y. Wang, T. E. Anderson, and D. A. Patterson. Cooperative Caching: Using Remote Client Memory to Improve File System Performance. In Proc. of ACM SIGMETRICS, 1994. 54, 64, 67

10. J. H. Howard. et.al. Scale and Performance in Distributed File Systems. ACM Transactions on Computer Systems, Feburary 1988. 64

11. R. M. Fujimoto and R. M. Weatherly. Time Management in the DoD High Level Architecture. In Proceedings of the Tenth Work-shop on Parallel and Distributed Simulations, pages 60-67, 1996. 67

12. S. Ghee. DVS: A Distributed VR Systems Infrastructure. In ACM SIGGRAPH Course Notes, 1995. 66

13. R. Gossweiler, R. J. Laferriere, M. L. Keller, and Paush. An Introductory Tutorial for Developing Multiuser Virtual Environments. Presence: Teleoperators and Virtual Environments, 3(4), 1990. 48

14. J. Gwertzman and M. Seltzer. World-Wide Web Cache Consistency. In Proc. of the 1996 USENIX Technical Conference, Jan 1996, 1996. 67

15. O. Hagsand. Interactive Multiuser VES in the DIVE System. IEEE Multimedia, 1996. 67

16. K. Li and P. Hudak. Memory Coherence in Shared Virtual Memory Systems. ACM Transactions on Computer Systems, 1989. 54

17. C. Liu and P. Cao. Maintaining Strong Consistency in the World-Wide Web. In Proc. of the International Conference on Distributed Computing Systems, 1997. 67

18. M. R. Macedonia, M. Z. Zyda, D. R. Pratt, P. T. Barham, and S. Zeswitz. NPSNET: A Network Software Architecture for Large-Scale Virtual Environments. Presence, 3(4):265-287, 1990. 67 
19. M. N. Nelson, B. B. Welch, and J. K. Osterhout. Caching in Sprite File Sytem. ACM Transactions on Computer Systems, 1988. 54, 64

20. P. Cao. A Collection of Web Proxy/Client Traces.

http://www.cs.wisc.edu/ cao/icache/proxytrace.html. 64

21. P. Cao. A Collection of Web Server Traces. http://www.cs.wisc.edu/ cao/icache/trace.html. 64

22. R. Sandberg, D. Boldberg, S. Kleiman, D. Walsh, and B.Lyon. Design and Implementation of Sun Network Filesystem. In Proc. of the Summer Usenix Conference, 1985. 54, 64

23. Borland Technologies. The VisigenicC ++ Programmer's Guide. http://www.visigenic.com/techpubs/books/vbj/vbj40/framesetindex.html. 67

24. Iona Technologies. The Orbix C++ Programmer's Guide. http://www.iona.com/products/orbix/manuals/index.html. 46, 67

25. M. van Steen, P. Homburg, and A. Tanenbaum. Globe: A Wide-Area Distributed System. IEEE Concurrency, pages 70-78, January-March 1999. 67

26. Q. Wang, M. Green, and C. Shaw. EM - An Environment Manager for Building Networked Virtual Environments. In Proceedings of IEEE VRAIS, 1995. 66

27. K. Worrell. Invalidation in Large Scale Network Object Caches. Master's thesis, University of Colarado, 1994. 67

28. J. Zinky, D. Bakken, and R. Schantz. Architectural Support for Quality of Service for CORBA Objects. In Theory and Practice of Object Systems, pages 41-49, 1997. $46,48,49$ 